\title{
A teenager with ductal carcinoma in situ arising in a fibroadenoma: a case report
}

\author{
Oliver Frisch, ${ }^{1}$ Louis Johann De Jager, ${ }^{2}$ Michael Otto, ${ }^{2}$ Jennifer Downs, ${ }^{1}$ Francois Malherbe ${ }^{1}$ \\ ${ }^{1}$ Division of General Surgery, Breast and Endocrine Surgery Unit, Groote Schuur Hospital, Faculty of Health Sciences, \\ University of Cape Town, Cape Town, South Africa \\ ${ }^{2}$ Department of Anatomical Pathology, National Health Laboratory Service and Faculty of Health Sciences, University \\ of Cape Town, Cape Town, South Africa
}

Corresponding author: Oliver Frisch (oliverffrisch@charite.de)

\begin{abstract}
Summary: Breast cancer arising within fibroadenomas (BcaFad) is a very rare finding. BcaFad occurs mostly in women between the ages of 40 and 50. This report details the unique case of an 18-year-old woman with bcafad. We present an 18-year-old woman with a ductal carcinoma in situ (DCIS) arising within a fibroadenoma. This is the youngest reported case of BcaFad in the literature. Clinically, the mass presented characteristics of a benign breast lump. Microscopically, an atypical proliferation of luminal epithelial cells was classified as a low-grade DCIS. As a very rare presentation at an especially unusual age, this case challenges the current risk evaluation and management in young women diagnosed with fibroadenoma.
\end{abstract}

Keywords: fibroadenoma, ductal carcinoma in situ, breast cancer, young age

S Afr J Surg 2018;56(4) http://dx.doi.org/10.17159/2078-5151/2018/v56n4a2766

\section{Introduction}

Fibroadenomas are the most common cause of palpable breast masses in young females with the majority occurring in the age group 20-40 years. ${ }^{1-3}$ However, breast cancer arising within fibroadenomas (BcaFad) is a very rare finding, with an incidence ranging between $0.002 \%$ and $0.125 \%{ }^{4}$ Carcinoma in situ (CIS) accounts for approximately $80 \%$ of diagnoses, far more common than invasive carcinomas (IC), which constitute $15 \%$ of the total carcinomas. ${ }^{5}$ According to various case reports, the mean age of patients diagnosed with BcaFad is in the fifth decade..$^{5-7}$ To the best of our knowledge, we present the youngest reported case of DCIS arising within a fibroadenoma in the English literature. Previously, DCIS was described arising within a fibroadenoma of a 30-year-old female..$^{4-6}$

\section{Case report}

An 18-year-old nulliparous woman presented with a history of right-breast tenderness and a palpable mass in the lower inner quadrant. She first noted the lump four to five years previously, and it was stable in size. She described herself as a healthy, active non-smoker. She was not using any form of hormonal contraception or other medication. There was no family history of breast cancer.

She was first seen in our clinic one year before eventual surgical excision of the mass. Examination revealed a clinically benign mass, $30 \times 20 \mathrm{~mm}$ in size, in the lower inner quadrant of the right breast. The mass was mobile, well-defined and not fixed to surrounding breast tissue and consistent with a fibroadenoma. Axillary examination was normal. An ultrasound supported the clinical findings of a benign lesion in the lower inner quadrant. In addition to the clinically palpable mass, a second retro-areolar lesion was found on ultrasound. The largest lesion measured $43 \mathrm{x}$ $24.5 \mathrm{~mm}$ and appeared multilobulated. The retro-areolar lesion measured 30.8 x $13.5 \mathrm{~mm}$ with a similar appearance. No intralesional vascularity or calcifications were reported. The patient was advised to undergo follow-up examinations to assess for interval change, in line with current unit protocol. At the next consultation six months later, the largest lump showed an increase in size with minor pain. Clinical examination revealed that the lump now measured $50 \times 50 \mathrm{~mm}$, but still had the characteristics of a benign breast lump. Surgery was advised because the lump increased in size and she underwent simple day case surgery, with no complications.

Both lesions were submitted to the Division of Anatomical Pathology for macroscopic and microscopic examination. Macroscopically, two fibrous nodules were received. The smaller nodule measured $40 \times 30 \times 28 \mathrm{~mm}$ and weighed $25.6 \mathrm{~g}$. The larger nodule measured $55 \times 48 \times 23 \mathrm{~mm}$ and weighed $32.7 \mathrm{~g}$. Microscopic examination of the smaller nodule showed a well-circumscribed, benign fibroepithelial lesion. A proliferative, cellular fibrous stroma was present 
around compressed ducts with an intracanalicular growth pattern. There was no evidence of malignancy and a diagnosis of juvenile fibroadenoma was favoured.

The larger nodule, corresponding to the mass which had lately increased in size, showed mostly similar histological
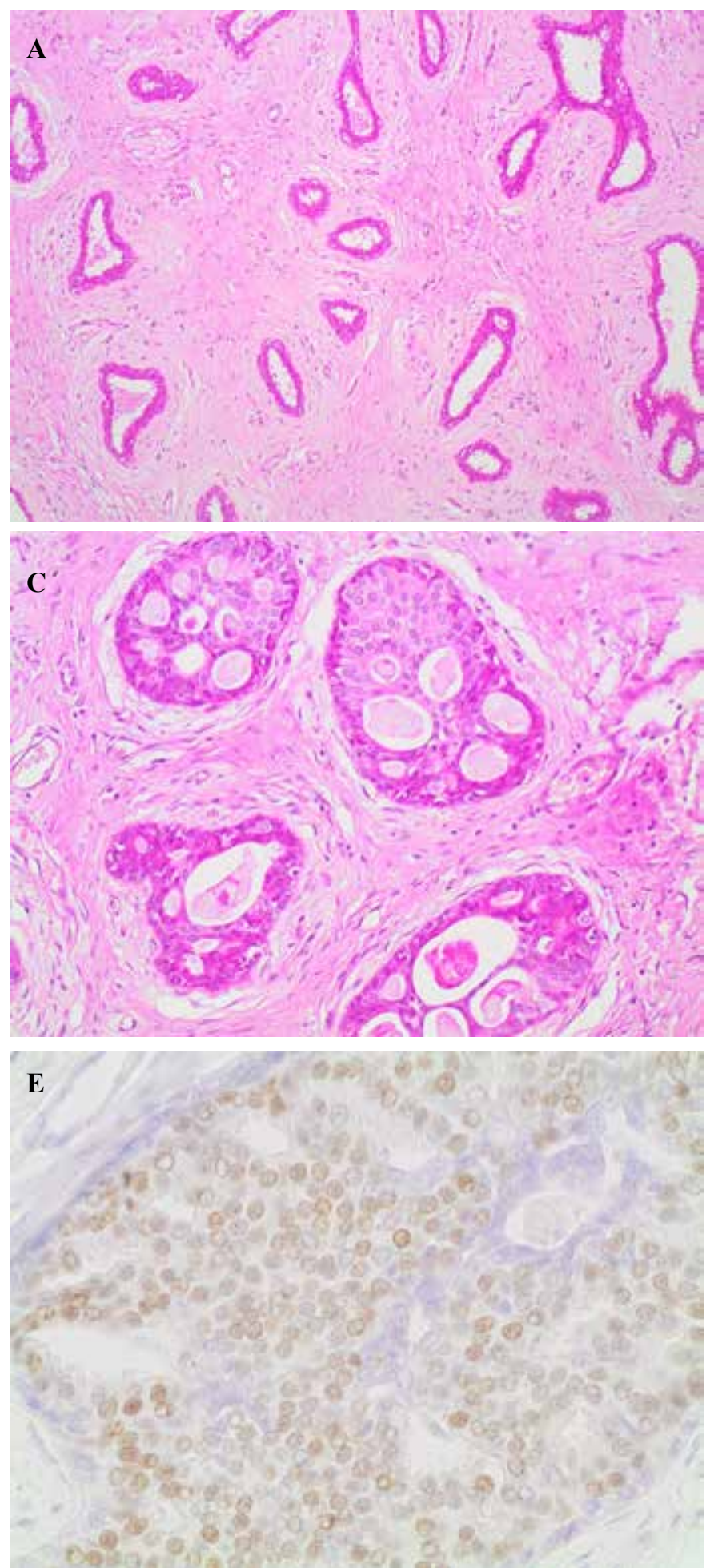

findings as compared to the smaller nodule. Sections showed a well-circumscribed, encapsulated biphasic lesion composed of compressed ducts with surrounding cellular fibrous stroma. However, focal ducts were expanded by an atypical proliferation of small, monomorphic luminal epithelial cells.
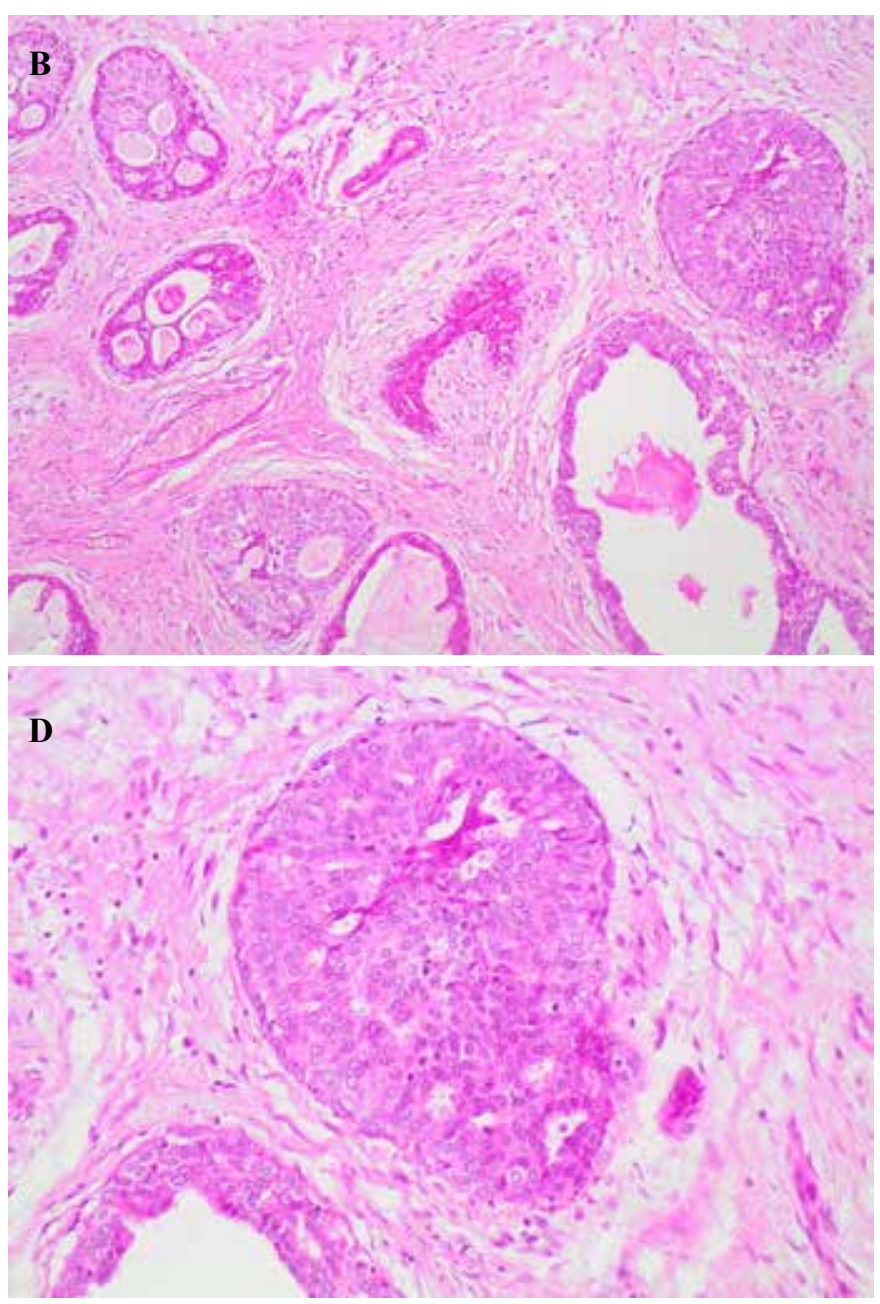

\section{F}

Figures. A) Fibroadenoma - benign ducts surrounded by fibrous stroma (H\&E x10 objective). B) Focus of low-grade ductal carcinoma in situ (H\&E x10 objective). C) Low-grade ductal carcinoma in situ - Ducts expanded by proliferation of atypical monomorphous epithelial cells with a pseudocribriform architecture (H\&E x20 objective). D) Low-grade ductal carcinoma in situ with a solid pattern (H\&E x20 objective). E) Strong diffuse Estrogen Receptor immunopositivity (x40 objective). F) Negative staining with high molecular weight cytokeratin markers (CK5/6 640 objective). 
A vague cribriform architecture was noted in these ducts. There was no intraductal necrosis present and there was no evidence of invasive malignancy. This focus measured $5 \mathrm{x}$ $2.5 \mathrm{~mm}$. Immunohistochemical (IHC) staining for CK5/6 showed no staining within the atypical epithelial proliferation, while ER staining demonstrated diffuse nuclear positivity of the atypical cells. The sample showed an Allred score of eight out of eight. ${ }^{8}$ In view of the morphologic features and IHC staining pattern, this proliferation was best classified as a focus of low-grade DCIS arising within a fibroadenoma. The closest surgical resection margin was $7.3 \mathrm{~mm}$. The patient scored seven out of 12 according to the University of Southern California/Van Nuys Prognostic Index (USC/VNPI) ( 1 for size, 1 for grade, 3 for age and 2 for margins).

Her surgical margins were clear and the decision was made not to do any further surgery or treat her with radiotherapy. After tumour board review, we decided not to offer her tamoxifen and rather obtain baseline magnetic resonance imaging (MRI) of her breast and to follow her up as a highrisk patient with yearly imaging studies and six monthly clinical breast examination.

\section{Discussion}

Fibroadenomas commonly occur in young females. It is controversial whether fibroadenomas increase the risk of developing breast cancer. Dupont et al. reported a risk of developing IC of up to 3.10 times higher in patients diagnosed with a complex fibroadenoma. ${ }^{9}$ However, another study ascribed the increased incidence of breast cancer within fibroadenomas to selection bias. ${ }^{10}$ Nevertheless, the rare occurrence of breast cancer developing within a fibroadenoma is estimated at an incidence of $0.002-0.125 \%$. The main type of malignancy in BcaFad is CIS, with $66.9 \%$ being lobular carcinomas in situ (LCIS) and $12.4 \%$ being DCIS. ${ }^{5}$ Of the $15 \%$ of carcinomas that are invasive, $11 \%$ are invasive ductal carcinomas (IDC) and $3.4 \%$ are invasive lobular carcinomas (ILC). ${ }^{4}$

There is no established care protocol for the management of fibroadenomas in teenagers. There is growing evidence that core biopsy is not routinely required in the adolescent as it is unlikely to affect patient management. In a retrospective study of 357 patients aged 25 years or less with a breast mass consistent with fibroadenoma (clinically and on ultrasound), $78.7 \%$ were histologically confirmed and $21.0 \%$ had another benign mass or normal histopathology (of whom $0.8 \%$ had a benign phyllodes tumour). Only $0.3 \%$ had breast carcinoma and retrospective review of the breast images did confirm an ill-defined mass suspicious for malignancy. ${ }^{11}$ There is a risk of iatrogenic injury in the developing breast and this may outweigh the benefits of surgery. The iatrogenic risk is especially relevant when the risk of malignancy is low and fibroadenomas often resolve over time. ${ }^{12}$ At the Groote Schuur breast unit we follow up young patients $(<25$ years $)$, after the initial diagnosis of a fibroadenoma, based on clinical examination and ultrasound. Only large or atypical fibroadenomas either on clinical examination or ultrasound will receive a core biopsy to confirm the diagnosis. Patients with a giant fibroadenoma $(>5 \mathrm{~cm}$ ) will be offered surgical removal of the lump.

In 2008, the National Comprehensive Cancer Network (NCCN) recommended excision alone as an acceptable treatment alternative for patients with DCIS. ${ }^{13}$ The clinical dilemma remains in deciding which group of patients need added radiotherapy. This dilemma is even more appropriate in a teenager with DCIS given the long-term side effects of radiotherapy.

Multivariate analysis has shown that certain factors are independent predictors of local recurrence in patients with DCIS treated with breast conservation. Van Nuys Classification predicts local recurrence using a combination of nuclear grade and necrosis. ${ }^{14}$ The Van Nuys Prognostic Index (VNPI) added size and margin width in 1996 and the USC/VNPI added age at diagnosis to the algorithm in 2002. ${ }^{15,16}$ The USC/VNPI was further fine-tuned in 2010 , by including different treatment recommendations for patients with margins between 3-10 mm if their USC/VNPI falls between 7-9. ${ }^{17}$ The European Society of Breast Cancer Specialists (EUSOMA) guidelines suggest that an acceptable rate of in-breast recurrence is $1-2 \%$ per year. ${ }^{18}$ Using this standard, the recommended management for individuals, which include our patient who scores 7 with margins more or equal to $3 \mathrm{~mm}$ on the fine-tuned USC/VNPI, is excision alone without radiotherapy.

Current National Comprehensive Cancer Network (NCCN) guidelines recommend ER testing for DCIS patients and consideration of tamoxifen for women with ER positive disease or who undergo breast-conserving surgery (BCS) without radiation. ${ }^{13}$ This is done to prevent the rate of recurrence because no mortality benefit for tamoxifen after DCIS has been conclusively demonstrated. ${ }^{19}$ There is no literature published documenting the long-term safety of tamoxifen if started in such a young patient. Based on this evidence as well as the expected side effects of tamoxifen, we decided not to use tamoxifen as a risk reducing medication in our patient.

\section{Conclusion}

The literature contains several reports describing patients with $\mathrm{BcaFad}$, which is a very rare diagnosis. Our patient presented with the very unusual combination of DCIS arising within a fibroadenoma at the young age of 18 years. Consequently, this case challenges the current risk evaluation and management in young women diagnosed with a fibroadenoma and demonstrates why all breast masses, regardless of the patient's age, should be treated with suspicion and excised if any atypical features or rapid increase in size are present. Breast cancer in teenagers is an extremely rare event and we would not recommend change in management protocols based on this one case. 


\section{REFERENCES}

1. Carty NJ, Carter C, Rubin C, Ravichandran D, Royle GT, Taylor I. Management of fibroadenoma of the breast. Annals of the Royal College of Surgeons of England. 1995;77(2):127-30.

2. Houssami N, Cheung MN, Dixon JM. Fibroadenoma of the breast. The Medical Journal of Australia. 2001;174(4):185-8.

3. Mg A D A, Bhoopal S, Ramanujam R. Benign breast diseases: experience at a teaching hospital in rural India. 2013; p. 73.

4. Wu YT, Chen ST, Chen CJ, Kuo YL, Tseng LM, Chen DR, et al. Breast cancer arising within fibroadenoma: collective analysis of case reports in the literature and hints on treatment policy. World J Surg Oncol. 2014;12:335.

5. Fukuda M, Nagao K, Nishimura R, Matsuda M, Baba K, Ueno $\mathrm{Y}$, et al. Carcinoma arising in fibroadenoma of the breast-a case report and review of the literature. Japanese J Surg. 1989;19(5):593-6.

6. Diaz NM, Palmer JO, McDivitt RW. Carcinoma arising within fibroadenomas of the breast. A clinicopathologic study of 105 patients. Am J Clin Pathol. 1991;95(5):614-22.

7. Deschenes L, Jacob S, Fabia J, Christen A. Beware of breast fibroadenomas in middle-aged women. Can J Surg. 1985;28(4):372-4.

8. Harvey JM, Clark GM, Osborne CK, Allred DC. Estrogen receptor status by immunohistochemistry is superior to the ligand-binding assay for predicting response to adjuvant endocrine therapy in breast cancer. J Clin Oncol: official journal of the American Society of Clinical Oncology. 1999;17(5):147481.

9. Dupont WD, Page DL, Parl FF, Vnencak-Jones CL, Plummer WD Jr, Rados MS, et al. Long-term risk of breast cancer in women with fibroadenoma. New Eng J Med. 1994;331(1):10-5.

10. Ciatto S, Bonardi R, Zappa M, Giorgi D. Risk of breast cancer subsequent to histological or clinical diagnosis of fibroadenoma-retrospective longitudinal study of 3938 cases. Ann Oncol: official journal of the European Society for Medical Oncology. 1997;8(3):297-300.

11. Smith GE, Burrows P. Ultrasound diagnosis of fibroadenoma - is biopsy always necessary? Clin Radiol. 2008;63(5):511-5; discussion 6-7.

12. Cerrato F, Labow BI. Diagnosis and management of fibroadenomas in the adolescent breast. Seminars in Plastic Surgery. 2013;27(1):23-5.

13. NCCN. National Comprehensive Cancer Network. Clinical Practice Guidelines in Oncology: Breast Cancer, version 1.2018. 2018.

14. Silverstein MJ, Poller DN, Waisman JR, Colburn WJ, Barth A, Gierson ED, et al. Prognostic classification of breast ductal carcinoma-in-situ. Lancet. 1995;345(8958):1154-7.

15. Silverstein MJ, Lagios MD, Craig PH, Waisman JR, Lewinsky BS, Colburn WJ, et al. A prognostic index for ductal carcinoma in situ of the breast. Cancer. 1996;77(11):2267-74.

16. Silverstein MJ. The University of Southern California/Van Nuys prognostic index for ductal carcinoma in situ of the breast. Am J Surg. 2003;186(4):337-43.

17. Silverstein MJ, Lagios MD. Choosing treatment for patients with ductal carcinoma in situ: fine tuning the University of Southern California/Van Nuys Prognostic Index. Journal of the National Cancer Institute Monographs. 2010;2010(41):193-6.

18. Rutgers EJ. Quality control in the locoregional treatment of breast cancer. Eur J Cancer (Oxford, England : 1990). 2001;37(4):447-53.

19. Staley H, McCallum I, Bruce J. Postoperative Tamoxifen for ductal carcinoma in situ: Cochrane systematic review and metaanalysis. Breast. 2014;23(5):546-51. 